01

\title{
K теории атомно-молекулярной конверсии в условиях бозе-эйнштейновской конденсации
}

\author{
() П.И. Хаджи, А.П. Зинган \\ Приднестровский государственный университет им. Т.Г. Шевченко, \\ МД-3300 Тирасполь, Молдавия \\ Институт прикладной фризики АНМ, \\ Кишинев, Молдавия \\ I e-mail: zingan.anna@mail.ru
}

(Поступило в Редакцию 16 мая 2016 г.)

В приближении среднего поля получена система нелинейных дифференциальных уравнений, описывающая динамику атомно-молекулярной конверсии под действием двух рамановских импульсов резонансного лазерного излучения произвольной формы. Детально изучена динамика системы при воздействии на нее гауссовых импульсов. Показана возможность существования различных режимов временной эволюции системы в зависимости от начальных условий.

DOI: 10.21883/JTF.2017.04.44305.1893

\section{1. Введение}

В последние годы достигнут существенный прогресс в экспериментальном и теоретическом исследовании свойств бозе-эйнштейновского конденсата (БЭК) атомов. В [1] показано, что исследование динамики связанных атомно-молекулярных конденсатов в условиях резонанса Фешбаха либо стимулированного рамановского связывания двух атомов в молекулу могут привести к когерентной суперхимии, в рамках которой возможно стимулирование химических реакций. Теория рамановского параметрического процесса с участием двух лазерных импульсов как единого (одноступенчатого) процесса в приближении среднего поля была предложена в [2-4]. В этих работах изучен процесс конверсии двух одинаковых атомов в гомоядерную молекулу в присутствии двух импульсов резонансного лазерного излучения. В [5] теоретически был изучен двухступенчатый процесс конверсии двух различных бозе-атомов в гетероядерную молекулу под действием двух импульсов лазерного излучения. Этот процесс также можно рассматривать как единый, поскольку общий уровень возбужденной молекулы оказывается незаселенным [2,3].

Относительно недавно наблюдалось связывание атомов в гомоядерные молекулы $\mathrm{K}_{2}$ [6], $\mathrm{Li}_{2}$ [7], $\mathrm{Cs}_{2}$ [8], $\mathrm{Na}_{2}$ [9], $\mathrm{Rb}_{2}$ [10]. Наибольший интерес вызывает наблюдение гетероядерных молекул. При этом наблюдалась атомно-молекулярная конверсия в Ферми-Ферми, Бозе-Бозе и Ферми-Бозе смесях атомов с образованием гетероядерных молекул ${ }^{6} \mathrm{Li}^{40} \mathrm{~K}[11],{ }^{6} \mathrm{Li}^{23} \mathrm{Na}[12]$, ${ }^{7} \mathrm{Li}{ }^{133} \mathrm{Cs}[13],{ }^{23} \mathrm{Na}^{133} \mathrm{Cs}[14],{ }^{85} \mathrm{Rb}^{133} \mathrm{Cs}[15],{ }^{39} \mathrm{~K}^{85} \mathrm{Rb}$ [16], ${ }^{40} \mathrm{~K}^{87} \mathrm{Rb}$ [17], ${ }^{41} \mathrm{~K}^{87} \mathrm{Rb}$ [18]. Большим успехом является наблюдение гомоядерного тетрамера $\mathrm{Cs}_{4}[19]$ и трехатомной гетероядерной молекулы ${ }^{87} \mathrm{Rb}^{40} \mathrm{~K}^{6} \mathrm{Li}$ [20]. Это обстоятельство свидетельствует о возможности образования и более сложных молекул в условиях бозеконденсации.

\section{2. Постановка задачи. Основные уравнения}

Изучим динамику процесса атомно-молекулярной конверсии под действием двух ультракоротких рамановских импульсов произвольной формы, которую формально можно изобразить в виде реакции $a+a+c_{1} \leftrightarrow b+c_{2}$, где символы $a$ и $b$ представляют атом и молекулу соответственно, а $c_{1}$ и $c_{2}-$ фотоны с частотами $\omega_{1}$ и $\omega_{2}$. Рассматриваемый процесс, в сущности, есть процесс оптической рамановской нутации в условиях атомномолекулярной конверсии, состоящий в периодическом изменении населенностей атомного и молекулярного состояний под действием двух рамановских импульсов когерентного лазерного излучения. Два свободных одинаковых атома, находящиеся в бозе-конденсате с полной энергией $2 \hbar \omega_{0}$, переходят в основное состояние гомоядерной молекулы с энергией $\hbar \Omega_{0}$ через виртуальное возбужденное молекулярное состояние с энергией $E_{u}$, одновременно поглощая и излучая кванты света с энергиями $\hbar \omega_{1}$ и $\hbar \omega_{2}$ соответственно (рис. 1$)$. Гамильтониан

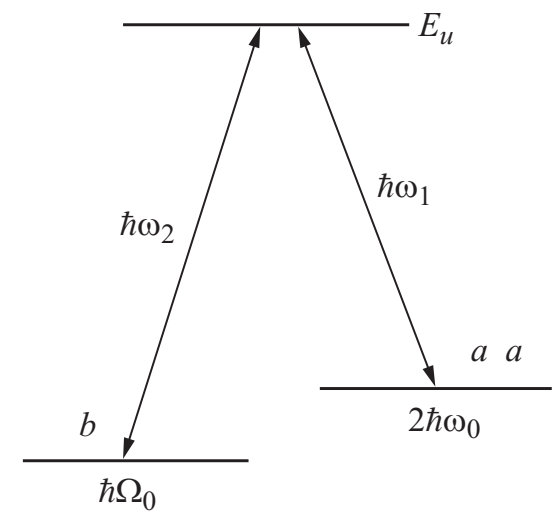

Рис. 1. Энергетическая схема и квантовые переходы в трехуровневой $\lambda$-схеме. 
взаимодействия $H_{\text {int }}$, описывающий процесс индуцированной атомно-молекулярной конверсии под действием двух ультракоротких импульсов лазерного излучения как единый (одноступенчатый) процесс, можно представить в виде

$$
H_{\mathrm{int}}=\hbar g\left(a^{+} a^{+} b c_{1}^{+} c_{2}+a a b^{+} c_{1} c_{2}^{+}\right),
$$

где $a, b$, и $c_{1,2}-$ бозонные операторы уничтожения атомов, молекул и фотонов соответственно, $g$ - константа взаимодействия. Нелинейность в (1) описывает образование гомоядерного молекулярного бозе-конденсата через стимулированную двумя рамановскими импульсами эмиссию молекулярных бозонов из атомного конденсата. Ранее гамильтониан (1) использовался для исследования процесса рамановской молекулярной фотоассоциации в предположении, что в начальный момент времени заданы плотности атомов, молекул и фотонов [5]. Было показано, что имеют место периодический и апериодический режимы атомно-молекулярной конверсии. При этом частоты колебаний плотностей частиц существенно зависят от начальных концентраций частиц. Однако эволюция системы под действием ультракоротких импульсов лазерного излучения произвольной формы не изучалась. Вместе с тем импульсное возбуждение системы с длительностями импульсов, на много меньшими времен релаксации атомов и молекул, является наиболее интересным, так как оно допускает возможность гибкого управления процессом атомно-молекулярной конверсии.

Используя (1), легко получить систему гайзенберговских уравнений для операторов $\hat{a}, \hat{b}$, и $\hat{c}_{1,2}$. Усредняя эту систему уравнений и используя приближение среднего поля (mean field approximation [21]), можно получить систему нелинейных дифференциальных уравнений для амплитуд (параметров порядка) материального $\langle\hat{a}\rangle=a,\langle\hat{b}\rangle=b$ и электромагнитного $\left\langle\hat{c}_{1,2}\right\rangle=c_{1,2}$ полей

$$
\begin{gathered}
i \dot{a}=\omega_{0} a+2 g a^{*} b c_{1}^{*} c_{2}, \\
i \dot{b}=\Omega_{0} b+g a a c_{1} c_{2}^{*}, \\
i \dot{c}_{1}=\omega_{1} c_{1}+g a^{*} a^{*} b c_{2}, \\
i \dot{c}_{2}=\omega_{2} c_{2}+g a a b^{*} c_{1},
\end{gathered}
$$

где $\dot{a}$ и т.д. означает производную по времени от функции $a(t)$ и т.д.

В условиях точного резонанса $\left(2 \omega_{0}-\Omega_{0}=\omega_{2}-\omega_{1}\right)$ решение уравнений (2) ищем в виде $a=A \times$ $\times \exp \left(-i \omega_{0} t+i \varphi\right), \quad b=B \exp \left(-i \Omega_{0} t+i \psi\right), \quad c_{1,2}=C_{1,2} \times$ $\times \exp \left(-i \omega_{1,2}+i \psi_{1,2}\right)$. В результате мы получаем новую систему нелинейных уравнений для амплитуд $A, B, C_{1,2}$ и разности фаз $\theta=2 \varphi-\psi+\psi_{1}+\psi_{2}$ :

$$
\begin{gathered}
\dot{A}=-2 g A B C_{1} C_{2} \sin \theta, \quad \dot{B}=g A^{2} C_{1} C_{2} \sin \theta \\
\dot{C}_{1}=-g A^{2} B C_{2} \sin \theta, \quad \dot{C}_{2}=g A^{2} B C_{1} \sin \theta \\
\dot{\theta}=g\left[-4 B C_{1} C_{2}+A^{2}\left(C_{1} C_{2} / B\right.\right. \\
\left.\left.-B C_{2} / C_{1}-B C_{1} / C_{2}\right)\right] \cos \theta
\end{gathered}
$$

Найдем решения системы (3)-(5), задавая следующие начальные условия: $A_{\mid t=0}=A_{0}=\sqrt{n_{0}}, B_{\mid t=0}=B_{0}=\sqrt{N_{0}}$, $\theta_{\mid t=0}=\theta_{0}$, где $n_{0}$ и $N_{0}$ - плотности атомов и молекул в начальный момент времени.

Рассмотрим сначала случай, когда оба импульса, падающие на систему атомов и молекул, являются ультракороткими. Амплитуды $c_{1}$ и $c_{2}$ полей этих импульсов будем считать заданными функциями времени и представим их в виде

$$
c_{1}=\sqrt{f_{10}} F_{1}(t), \quad c_{2}=\sqrt{f_{20}} F_{2}(t),
$$

где $F_{1}(t)$ и $F_{2}(t)$ - огибающие этих импульсов, а $f_{10}$ и $f_{20}$ - плотности фотонов в максимумах первого и второго импульсов. При этом мы считаем, что $f_{10}, f_{20} \gg n_{0}, N_{0}$, т. е. рассматриваем эволюцию системы в приближении заданных плотностей фотонов обоих импульсов. В этом приближении удается получить аналитические решения уравнений для плотностей атомов и молекул и разности фаз $\theta$. Для этого вместо времени введем переменную $\tau$, которая определяется интегралом

$$
\tau=g \sqrt{f_{10} f_{20}} \int_{-\infty}^{t} F_{1}\left(t^{\prime}\right) F_{2}\left(t^{\prime}\right) d t^{\prime} .
$$

Переменная $\tau(t)$ аналогична площади импульса, которая вводится при исследовании эволюции системы двухуровневых атомов под действием поля электромагнитной волны [22,23]. Однако в (7) под знаком интеграла содержится произведение огибающих обоих импульсов. Функция $\tau(t)$ является конечной для ограниченных во времени рамановских импульсов и существенно определяется степенью перекрытия их огибающих. Считая эти импульсы, например, гауссовыми: $F_{1}(t)=\exp \left(-t^{2} / \tau_{1}^{2}\right)$, $F_{2}(t)=\exp \left(-\left(t-t_{0}\right)^{2} / \tau_{2}^{2}\right)$, где $\tau_{1}$ и $\tau_{2}-$ полуширины этих импульсов, а $t_{0}-$ временная задержка между пиками обоих импульсов, для функции $\tau(t)$ получаем выражение

$$
\begin{aligned}
& \tau(t)=\frac{\sqrt{\pi}}{2} g \sqrt{f_{10} f_{20}} \tau_{1} \tau_{2} / \sqrt{\tau_{1}^{2}+\tau_{2}^{2}} \exp \left(-t_{0}^{2} /\left(\tau_{1}^{2}+\tau_{2}^{2}\right)\right) \\
& \times\left[1+\Phi\left(t \sqrt{\tau_{1}^{2}+\tau_{2}^{2}} / \tau_{1} \tau_{2}-t_{0} \tau_{1} /\left(\tau_{2} \sqrt{\tau_{1}^{2}+\tau_{2}^{2}}\right)\right)\right]
\end{aligned}
$$

где $\Phi(x)$ - функция вероятности. Переходя в (3)-(5) от $t$ к $\tau$, получаем интеграл движения для плотностей атомов и молекул

$$
n+2 N=n_{0}+2 N_{0},
$$

выражение для разности фаз через плотность частиц

$$
\theta=\arccos \left(\frac{n_{0}}{n} \sqrt{\frac{N_{0}}{N}} \cos \left(\theta_{0}\right)\right)
$$

и нелинейное дифференциальное уравнение, описывающее эволюцию плотности молекул

$$
\frac{d N}{d \tau}= \pm 2 \sqrt{N\left(n_{0}+2 N_{0}-2 N\right)^{2}-N_{0} n_{0}^{2} \cos ^{2} \theta_{0}} .
$$




\section{Обсуждение результатов}

Найдем решения уравнения (11) при различных значениях начальной разности фаз $\theta_{0}$. В случае если $\theta_{0}=\pi / 2$, то решение уравнения (11) имеет вид

$$
\begin{aligned}
N= & \left(N_{0}+\frac{n_{0}}{2}\right)\left(\left(\sqrt{N_{0}} \pm \sqrt{N_{0}+n_{0} / 2}\right.\right. \\
& \left.\times \operatorname{th} \sqrt{2\left(2 N_{0}+n_{0}\right) \tau}\right) /\left(\sqrt{N_{0}+n_{0} / 2}\right. \\
& \left.\left. \pm \sqrt{N_{0}} \operatorname{th} \sqrt{2\left(2 N_{0}+n_{0}\right)} \tau\right)\right)^{2} .
\end{aligned}
$$

Знаки „+““ и „-““ в (12) определяют направление начальной скорости изменения функции $N(t)$, т. е. $(d N / d \tau)_{\mid t=0}$. Из (12) следует, что при $n_{0}=0$ плотность молекул в любой момент времени $N=N_{0}=$ const. Следовательно, процесс распада молекул при $n_{0}=0$ с образованием свободных атомов не имеет места. Это обусловлено тем, что в отсутствие атомов в начальный момент времени отсутствует атомное стимулирование процесса и тогда, хотя плотности фотонов обоих импульсов и плотность молекул отличны от нуля, тем не менее система не эволюционирует. Отсюда также следует, что если в других условиях эволюция системы имеет место, но в какой-то момент времени плотность атомов обращается в нуль, то процесс нетривиальной эволюции на этом заканчивается.

Из (12) видно, что решение со знаком „,+“ монотонно растет с ростом переменной $\tau$ и асимптотически стремится к значению $N=N_{0}+\frac{n_{0}}{2}$ при $\tau \rightarrow \infty$ (рис. 2). Однако функция $\tau(t)$ ограничена сверху. Поэтому плотность молекул растет до некоторого предельного значения $N_{\lim }<N_{0}+n_{0} / 2$, которое определяется максимальным значением переменной $\tau$. Это означает, что не все атомы успевают связаться попарно в молекулы за время действия импульсов и часть атомов сохраняется в системе. В [5] было показано, что при $t \rightarrow \infty$ все исходные атомы превращаются в молекулы, чем эволюция и заканчивается. Однако в данном случае переменная $\tau$ не обращается в бесконечность и именно по этой причине предельная плотность молекул меньше $N_{0}+n_{0} / 2$. Но в то же время сохранившиеся атомы не стимулируют
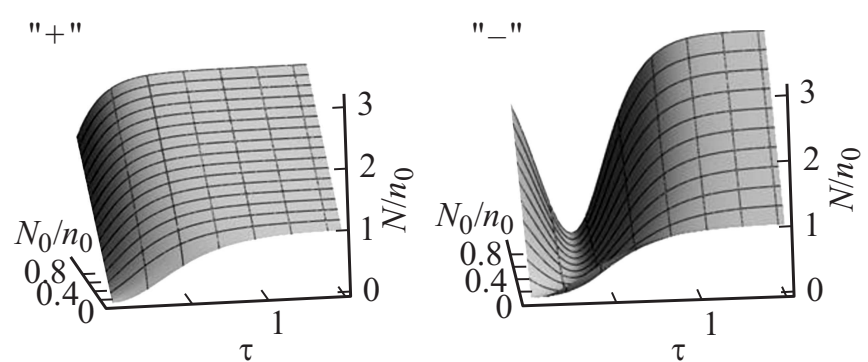

Рис. 2. Зависимость нормированной плотности молекул $N / n_{0}$ от функции $\tau(t)$ и нормированной начальной плотности молекул $N_{0} / n_{0}$ при $\theta_{0}=\pi / 2, n_{0}=2 \cdot 10^{14} \mathrm{~cm}^{-3}$, $\tau_{1}=\tau_{2}=2 \cdot 10^{-12} \mathrm{~s}, t_{0}=0, f_{10} / n_{0}=f_{20} / n_{0}=5$.

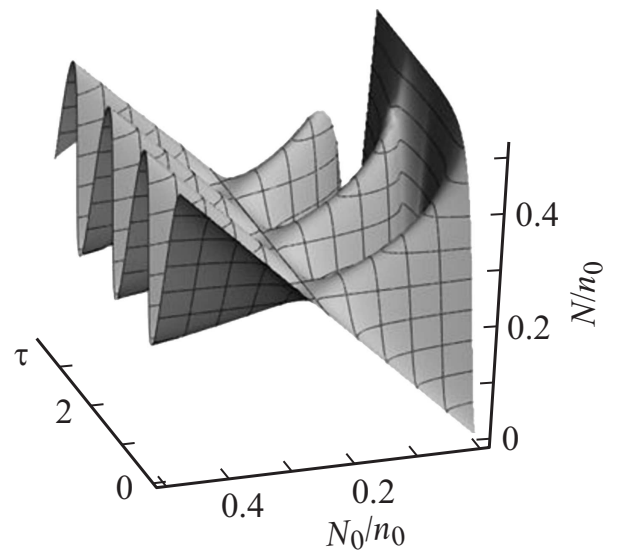

Рис. 3. Зависимость нормированной плотности молекул $N / n_{0}$ от функции $\tau(t)$ и нормированной начальной плотности молекул $N_{0} / n_{0}$ при $\theta_{0}=0, n_{0}=2 \cdot 10^{14} \mathrm{~cm}^{-3}, \tau_{1}=\tau_{2}=2 \cdot 10^{-12} \mathrm{~s}$, $t_{0}=0, f_{10} / n_{0}=f_{20} / n_{0}=5$.

систему, так как рост переменной $\tau$ заканчивается, как только оба импульса прошли через нее. В этом случае отсутствует стимулирование процесса фотонами падающих импульсов. Таким образом, процесс атомномолекулярной конверсии при $n_{0}=0$ является необратимым, так как учитываются только индуцированные переходы. Отметим, что если площадь перекрытия обоих импульсов $\tau=\tau_{\text {cr, }}$, то в этом случае после индуцированного распада начального количества молекул система перестает эволюционировать, т.е. плотности частиц перестают изменяться со временем.

Решение со знаком „-“ на начальном этапе монотонно убывает из-за индуцированного распада молекул (рис. 2). При $\tau=\tau_{\mathrm{cr}}=\operatorname{arth} \sqrt{N_{0} /\left(N_{0}+n_{0} / 2\right)} /$ $/ \sqrt{2\left(2 N_{0}+n_{0}\right)}$ плотность молекул обращается в нуль и в системе имеются только атомы с плотностью $2 N_{0}+n_{0}$. Затем с ростом $\tau$ плотность молекул начинает расти и при больших $\tau \gg \tau_{\text {cr }}$ в системе генерируется то же предельное значение молекул, что и в случае решения со знаком „+““. И в этом случае процесс конверсии является необратимым.

Если в начальный момент времени в системе отсутствуют молекулы $\left(N_{0}=0\right)$, то из (12) следует, что

$$
N=\frac{n_{0}}{2} \operatorname{th}^{2}\left(\sqrt{2 n_{0}} \tau\right)
$$

Таким образом, видно, что при $\theta_{0}=\pi / 2$ либо $\theta_{0}=$ $=(2 k+1) \pi / 2(k=0,1,2, \ldots)$ эволюция системы является апериодической. При этом часть атомов превращается в молекулы, чем эволюция и завершается. Предельное значение плотности молекул определяется начальными плотностями атомов, молекул и фотонов обоих импульсов, а также полуширинами импульсов и временем задержки между ними.

Рассмотрим теперь эволюцию системы при $\theta_{0}=0$ либо $\pi$. Тогда решение уравнения (11) выражается 

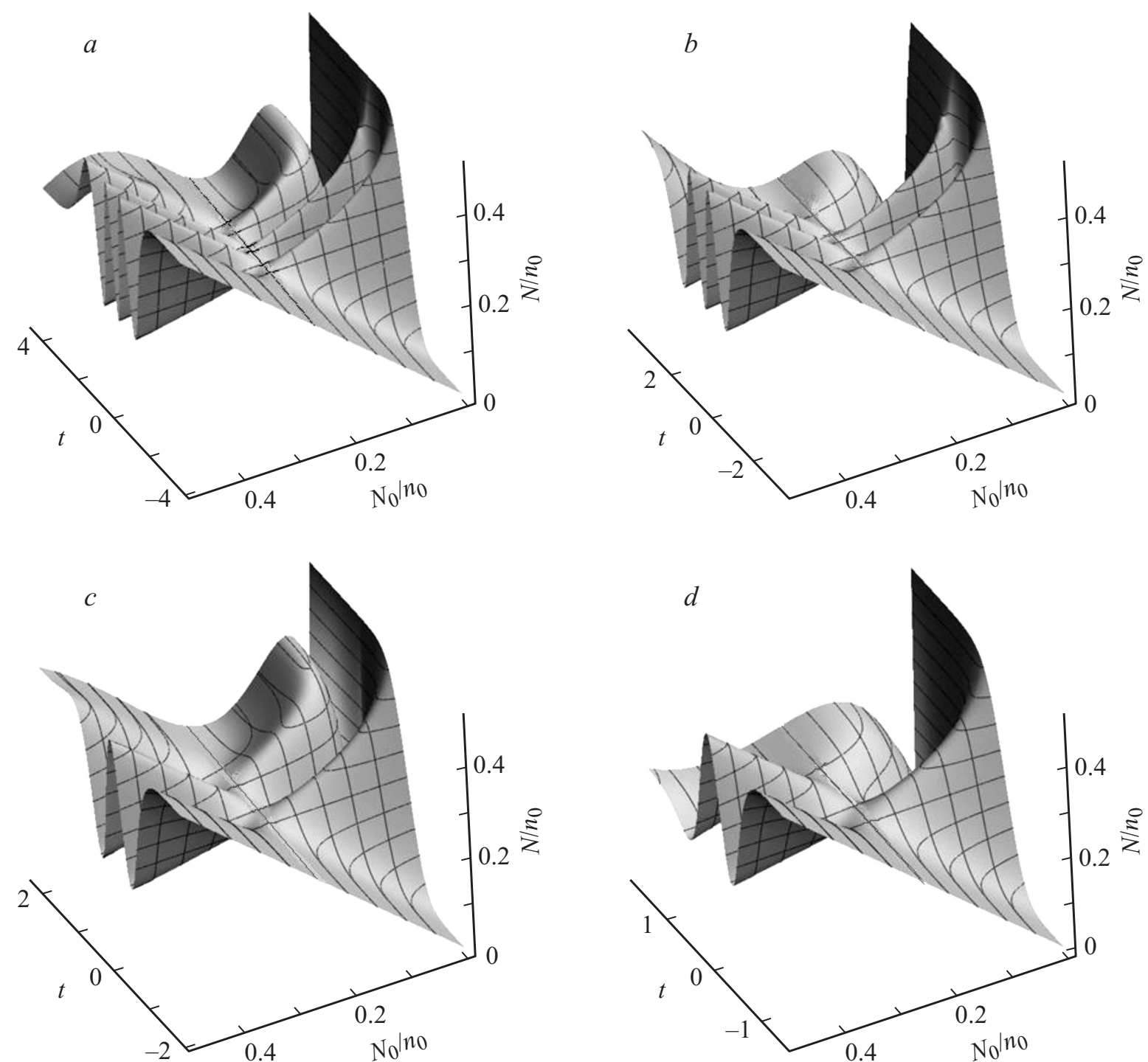

Рис. 4. Временная эволюция нормированной плотности молекул $N / n_{0}$ в зависимости от нормированной начальной плотности молекул $N_{0} / n_{0}$ при $n_{0}=2 \cdot 10^{14} \mathrm{~cm}^{-3}, f_{10} / n_{0}=f_{20} / n_{0}=5 ; a-\tau_{1}=\tau_{2}=2 \cdot 10^{-12} \mathrm{~s}, t_{0}=0 ; b-\tau_{1}=\tau_{2}=2 \cdot 10^{-12} \mathrm{~s}, t_{0}=1 \cdot 10^{-12} \mathrm{~s}$; $c-\tau_{1}=1 \cdot 10^{-12} \mathrm{~s}, \tau_{2}=2 \cdot 10^{-12} \mathrm{~s}, t_{0}=0 ; d-\tau_{1}=\tau_{2}=1 \cdot 10^{-12} \mathrm{~s}, t_{0}=0$.

формулами:

$$
\begin{gathered}
N=N_{0}+\left(N_{-}-N_{0}\right) \operatorname{sn}^{2}\left(2 \sqrt{N_{+}-N_{0}} \tau\right), \\
k^{2}=\left(N_{-}-N_{0}\right) /\left(N_{+}-N_{0}\right)
\end{gathered}
$$

при $N_{0}<n_{0} / 4$,

$$
\begin{gathered}
N=N_{+}-\left(N_{+}-N_{0}\right) / \operatorname{dn}^{2}\left(2 \sqrt{N_{+}-N_{-}} \tau\right), \\
k^{2}=\left(N_{0}-N_{-}\right) /\left(N_{+}-N_{-}\right)
\end{gathered}
$$

при $N_{0}>n_{0} / 4$ и

$$
N=N_{-}=N_{0}=\text { const }
$$

при $N_{0}=n_{0} / 4$, где $\operatorname{sn} x, \operatorname{dn} x-$ эллиптические функции Якоби, $k$ - модуль эллиптических функций, а $N_{+}, N_{-}$ и $N_{0}$ - корни уравнения

$$
N\left(n_{0}+2 N_{0}-2 N\right)^{2}-N_{0} n_{0}^{2} \cos ^{2} \theta_{0}=0,
$$

причем $N_{+}>N_{-}>N_{0}$. На рис. 3 представлена зависимость плотности молекул от переменной $\tau$ при различных значениях параметра $N_{0} / n_{0}$. Видно, что с ростом $\tau$ плотность молекул эволюционирует и в зависимости от полной площади перекрытия импульсов имеет место периодическая эволюция системы с ограниченным числом периодов. При $N_{0}<n_{0} / 4$ плотность молекул колеблется над фоном, а при $N_{0}>n_{0} / 4-$ под фоном, равным начальной плотности молекул $N_{0}$. При этом минимум (максимум) функции $N(\tau)$ при $N_{0}<n_{0} / 4$ переходит в максимум (минимум) при $N_{0}>n_{0} / 4$, а при $N_{0}=n_{0} / 4$ она остается постоянной, равной $N_{0}$. В этом случае хотя начальные плотности атомов и молекул отличны от нуля, тем не менее система не изменяет своих показателей со временем, т. е. она покоится. Таким образом, можно отметить, что важной особенностью 
временной эволюции атомов и молекул является то обстоятельство, что при начальной разности фаз $\theta_{0}=0, \pi$ $(n \pi, n=0,1,2, \ldots)$ амплитуда осцилляций плотности молекул обращается в нуль при определенном соотношении между параметрами. Кроме того, значения площадей $\tau$, для которых имеет место нетривиальная эволюция, ограничены сверху и являются различными для различных значений амплитуд $f_{10}$ и $f_{20}$ и длительностей $\tau_{1}$ и $\tau_{2}$ импульсов. При фиксированном $\tau$ число периодов колебаний плотности молекул растет с ростом параметра $N_{0} / n_{0}$.

На рис. 4, a представлена зависимость плотности молекул от текущего времени $t$ для случая, когда на систему падают два гауссовых импульса с длительностями $\tau_{1}$ и $\tau_{2}$ в отсутствие задержки $\left(t_{0}=0\right)$. Видно, что на начальном этапе с ростом времени плотность молекул практически не изменяется и равна начальной плотности $N_{0}$, так как площадь перекрытия импульсов на их передних хвостах практически равна нулю и слабо растет. В окрестности пиков импульсов (при $t \cong 0$ ) площадь перекрытия $\tau(t)$ быстро растет, что приводит к колебательному изменению плотности молекул. С дальнейшим ростом времени (на задних хвостах импульсов) площадь перекрытия практически перестает изменяться и функция $N(t)$ перестает осциллировать. Именно это значение предельной площади перекрытия $\tau(t)$ определяет предельный уровень плотности молекул, которые генерируются за время действия обоих импульсов. Иными словами, этим определяется уровень производства молекул при заданных огибающих рамановских импульсов. Отметим, что, как следует из рис. 4, $a$, предельные значения плотности молекул отличаются от начальных значений и определяются параметром $N_{0} / n_{0}$. Таким образом, выбирая определенные значения начальных плотностей атомов и молекул, можно управлять значением плотности образующихся молекул на заключительной стадии протекания реакции.

Из рис. 4 видно, что увеличение времени задержки $t_{0}$ (рис. 4,b), дисбаланс полуширин импульсов (рис. $4, c)$, уменьшение полуширин (и амплитуд) импульсов (рис. 4,d) приводит к уменьшению общей площади перекрытия $\tau$ импульсов и к изменению особенностей временной эволюции системы.

Из анализа результатов, полученных для $\theta_{0}=0, \pi$, $\pi / 2$, можно сделать вывод, что закономерности процесса стимулированной атомно-молекулярной конверсии определяются также и начальной разностью фаз $\theta_{0}$ наряду с начальными плотностями частиц. Из (11) следует, что особенности эволюции определяются корнями уравнения (17). При произвольных $\theta_{0}$ это уравнение имеет три действительных корня, которые мы представим в порядке их возрастания в виде $N_{m}<N_{M}<N_{1}$, где $N_{M}$ и $N_{m}$ - максимальное и минимальное значения плотности молекул в процессе эволюции. Тогда решение

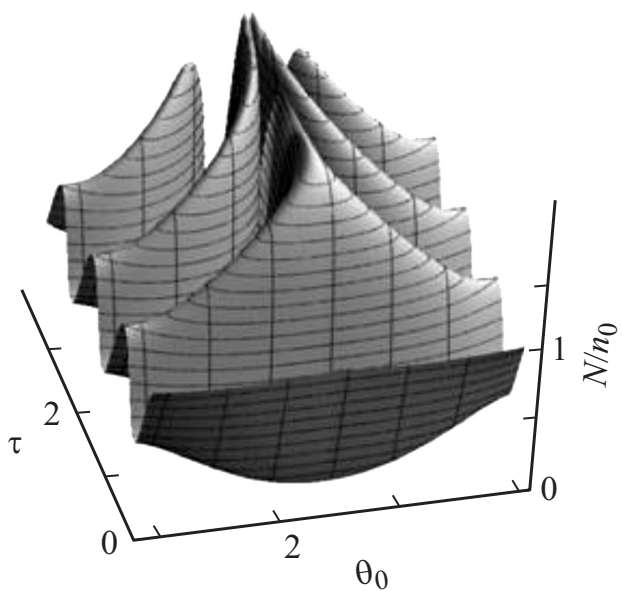

Рис. 5. Зависимость нормированной плотности молекул $N / n_{0}$ от функции $\tau(t)$ и начальной разности фаз $\theta_{0}$ при $n_{0}=2 \cdot 10^{14} \mathrm{~cm}^{-3}, \tau_{1}=\tau_{2}=2 \cdot 10^{-12} \mathrm{~s}, t_{0}=0, f_{10} / n_{0}=$ $=f_{20} / n_{0}=5$.

уравнения (11) выражается формулой

$$
N=N_{m}+\left(N_{M}-N_{m}\right) \operatorname{sn}^{2}\left(2 \sqrt{N_{1}-N_{m}} \tau \pm F\left(\varphi_{0}, k\right)\right),
$$

где

$$
\begin{gathered}
\varphi_{0}=\arcsin \sqrt{\left(N_{0}-N_{m}\right) /\left(N_{M}-N_{m}\right)}, \\
k^{2}=\left(N_{M}-N_{m}\right) /\left(N_{1}-N_{m}\right),
\end{gathered}
$$

$F\left(\varphi_{0}, k\right)$ - неполный эллиптический интеграл первого рода с модулем $k$ и параметром $\varphi_{0}$.

На рис. 5 представлена временная эволюция плотности молекул $N(t)$ для различных значений параметра $\theta_{0}$ при фиксированных значениях $N_{0}$ и $n_{0}$. При фиксированном $t$ плотность молекул зависит от $N_{0}$ и $n_{0}$, от начальной разности фаз $\theta_{0}$, а также от текущего значения площади перекрытия импульсов $\tau$. Отметим, что функция $N(\tau)$ является периодической, тогда как функция $N(t)$ является колебательной непериодической. Из рис. 5 видно, что с ростом времени функция $N(t)$ испытывает несколько колебаний, число которых определяется площадью перекрытия импульсов $\tau$, после чего колебательный режим эволюции прекращается, плотность молекул перестает изменяться, причем предельная плотность молекул после окончания действия импульсов определяется максимальным значением площади перекрытия.

Отметим, что если ширина одного из импульсов на много меньше ширины другого, причем узкий импульс действует на фоне широкого, то, как следует из (7), эффективность процесса определяется площадью узкого импульса и амплитудой широкого.

Пусть длительность одного из импульсов на много превосходит длительность другого $\left(\tau_{1} \gg \tau_{2}\right.$ либо $\left.\tau_{2} \gg \tau_{1}\right)$. В этом случае решения (9)-(19) для плотности молекул в приближении заданных плотностей фотонов обоих импульсов $\left(f_{10}, f_{20} \gg N_{0}, n_{0}\right)$ сохраняются, 
но площадь перекрытия импульсов $\tau$ теперь определяется выражением

$$
\tau=(\sqrt{\pi} / 2) g \tau_{0} \sqrt{f_{10} f_{20}}\left[1+\Phi\left(t / \tau_{0}\right)\right],
$$

где $\tau_{0}=\min \left(\tau_{1}, \tau_{2}\right)$ - меньшая из длительностей импульсов $\tau_{1}$ и $\tau_{2}$. По сути дела множитель $1+\Phi\left(t / \tau_{0}\right)$ определяет площадь узкого импульса, так как области, где амплитуда этого импульса исчезающе мала, практически не вносят вклада в интеграл (7). В этом случае поведение функции $N(t)$ качественно такое же, как и в случае сравнимых длительностей импульсов $\tau_{2} \cong \tau_{1}$. Плотность молекул испытывает колебания в течение времени, пока действует узкий импульс, после чего плотность молекул перестает изменяться. Предельная плотность образующихся молекул определяется значением параметра $\tau$.

\section{Заключение}

В заключение отметим, что в настоящей работе представлены результаты исследования явления атомномолекулярной конверсии под действием двух ультракоротких рамановских импульсов резонансного лазерного излучения. В приближении заданной плотности фотонов обоих импульсов, имеющих произвольную форму, удается получить точные аналитические решения для плотности образующихся молекул путем введения площади перекрытия обоих импульсов либо площади одного из них. Показано, что эволюция системы может быть как периодической, так и апериодической. Предсказана возможность контроля за ходом реакции и уровнем производства молекул вариацией площадей падающих импульсов и начальных плотностей материальных частиц.

\section{Список литературы}

[1] Krems R.V., Stwalley W.C., Friedrich B. Cold Molecules: Theory, Experiment, Applications. Boca Raton, London, NY: CRC Press, Taylor and Francis Group, 2009. 753 p.

[2] Хаджи П.И., Ткаченко Д.В. // Письма в ЖЭТФ. 2006. T. 83. C. 120.

[3] Хаджи П.И., Ткаченко Д.В. // ЖЭТФ. 2007. Т. 131. С. 425.

[4] Khadzhi P.I., Tkachenko D.V. // J. Nanoelectron. Optoelectron. 2009. Vol. 4. P. 101.

[5] Хаджси П.И., Зинган А.П. // Письма в ЖЭТФ. 2010. Т. 92. C. 490.

[6] Regal C.A., Ticknor G., Bohn J.L., Jin D.S. // Nature. 2003. Vol. 424. P. 47.

[7] Strecker K.E., Partridge G.B., Hulet R.G. // Phys. Rev. Lett. 2003. Vol. 91. P. 080406.

[8] Herbig J., Kraemer T., Mark M., Weber T., Chin C., Nägerl H.-C., Grimm R. // Science. 2003. Vol. 301. P. 1510.

[9] Devis K.B., Mewes M.-O., Andrews M.R., van Druten N.J., Durfee D.S., Kurn D.M., Ketterle W. // Phys. Rev. Lett. 1995. Vol. 75. P. 3969.
[10] Courteile Ph., Freeland R.S., Heinzen D.J., van Abeelen F.A., Verhaar B.J. // Phys. Rev. Lett. 1998. Vol. 81. P. 69.

[11] Voigt A.-C., Taglieber M., Costa L., Aoki T., Wieser W., Hänsch T.W., Dieckmann K. // Phys. Rev. Lett. 2009. Vol. 102. P. 020405.

[12] Stan C.A., Zwierlein M.W., Schunck C.H., Raupach S.M.F., Ketterle W. // Phys. Rev. Lett. 2004. Vol. 93. P. 143001.

[13] Soderberg K.-A.B., Gemelke N., Chin C. // New J. Phys. 2009. Vol. 11. P. 055022.

[14] Haimberger C., Kleinert J., Bhattacharya M., Bigelow N.P. // Phys. Rev. A. 2004. Vol. 70. P. 021 402(R).

[15] Sage J.M., Sainis S., Bergeman T., De Mille D. // Phys. Rev. Lett. 2005. Vol. 94. P. 203001.

[16] Kim J.T., Wang D., Eyler E.E., Gould P.L., Stwalley W.C. // New J. Phys. 2009. Vol. 11. P. 055020.

[17] Ospelkaus S., Pe'er A., Ni K.-K., Zirbel J.J., Neyenhuis B., Kotochigova S., Julienne P.S., Ye J., Jin D.S. // Nature Phys. 2008. Vol. 4. P. 622.

[18] Aikawa K., Akamatsu D., Kobayashi J., Ueda M., Kishimoto T., Inouye S. // New J. Phys. 2009. Vol. 11. P. 055035.

[19] Chin C., Kraemer T., Mark M., Herbig J., Waldburger P., Nägerl H.-C., Grimm R. // Phys. Rev. Lett. 2005. Vol. 94. P. 123201.

[20] Taglieber M., Voight A.-C., Aoki T., Hänsch T.W., Dieckmann K. // Phys. Rev. Lett. 2008. Vol. 100. P. 010401.

[21] Khadzhi P.I., Tkachenko D.V. // J. Nanoelectron. Optoelectron. 2009. Vol. 4. P. 101.

[22] Аллен Л., Эберли Дж. // Оптический резонанс и двухуровневые атомы. М.: Мир, 1978. 222 с.

[23] Скалли М.О., Зубайри М.С. // Квантовая оптика. М.: Физматлит, 2003. $511 \mathrm{c.}$ 